\title{
Hepatosplenic Gamma Delta T-cell Lymphoma Presenting as Pro- gressive Hepatosplenomegaly Highlighted during Pregnancy
}

\author{
Hussam Almasri, MD ${ }^{1^{*}}$ (D) Mahir Petkar, MBBS, MD, FRCPath ${ }^{2}$, Abdulaziz Zafar, MBBS, MRCP1, Mo- \\ hammed Gaber, $M D^{1}{ }^{(D}$, Mohammad Altermanini, $M B B C H^{1}$, Rita Ahmad, $M D^{1}$, and Mouhammad \\ Sharaf Eldean, MD, FCAP2
}

${ }^{1}$ Department of Medicine, Hamad Medical Corporation, Qatar

${ }^{2}$ Department of Pathology and Laboratory Medicine, Hamad Medical Corporation, Qatar

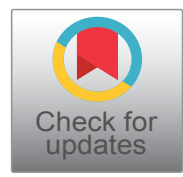

*Corresponding author: Hussam Almasri, MD, Department of Medicine, Hamad General Hospital, PO Box 3050, Doha, Qatar, Tel: +9745593346

\begin{abstract}
Hepatosplenic T-cell lymphoma (HSGDTL) is a rare variant of T-cell lymphoma with few reported cases in literature. It usually presents with hepatosplenomegaly and constitutional symptoms, primarily in young to middle aged patients. The prognosis is generally poor, with maximum reported survival periods of 2-3 years. There is a link to immunodeficiency and some biological treatments. Liver biopsy with flow cytometry is required to confirm the diagnosis.

We report a case of gamma delta $(\gamma \delta) \mathrm{HSTCL}$ in a 37-yearold female presenting as progressive hepatosplenomegaly with cytopenia during pregnancy. The patient underwent chemotherapy, splenectomy and bone marrow transplant and is in remission 10 months after the diagnosis.

As far as we are aware, this is the first case of $\gamma \delta$ HSGDTL reported in the Middle East and the second documented case of HSGDTL that highlighted during pregnancy.
\end{abstract}

\section{Keywords}

Hepatosplenic T-cell lymphoma, Gamma delta T-cell lymphoma, Pregnancy

\section{Introduction}

Hepatosplenic gamma delta $(\gamma \delta)$ T-cell lymphoma (HSGDTL) is a rarely encountered peripheral T-cell lymphoma. It accounts for less than 1 percent of all Non-Hodgkin Lymphomas [1]. Prognosis is poor, although data is limited because of its relative rarity. Patients are usually young to middle aged males, $30-40$ years-old. Less than 20 percent of cases show possible link to immunosuppression such as organ transplant and inflammatory bowel disease patients exposed to immunomodulators [2].

The underlying mechanism of this disorder is unknown, although association with isochromosome $7 q$ has been reported [3]. Most HSTCL have the gamma-delta subtype of the T-cell receptors (TCR) with few cases reported to have the alpha-beta TCR subtype [4].

As the name suggests, HSGDTL presents with hepatosplenic involvement, causing massive hepatosplenomegaly. Lymphadenopathy is not common. Patients have pancytopenia or at times, only thrombocytopenia. Constitutional symptoms are varied and most cases are also reported to have bone marrow involvement.

Chemotherapy is the mainstay of treatment, but the outcome is generally poor. Splenectomy has been reported to be beneficial in patients with thrombocytopenia, prior to the start of chemotherapy [5].

To our knowledge, only one report has documented the development of HSGDTL during pregnancy. This was diagnosed post-splenectomy with remission achieved after chemotherapy [6]. Another case was reported to have developed the disease during the post-partum period, with an otherwise healthy and uneventful pregnancy [7].

Herein, we describe a case of HSGDTL, presenting with progressive marked hepatosplenomegaly and pancytopenia during early pregnancy, the splenomegaly 
was noted just before pregnancy initiation as the patient presented with vague abdominal pain and constipation, during pregnancy hepatosplenomegaly and pancytopenia became more evident and diagnosis was confirmed with liver biopsy after delivery.

\section{Case Report}

A 37-year-old Egyptian female with no significant medical history presented with vague abdominal pain and constipation, 1 month before pregnancy. Ultrasound revealed spleen size of $16.3 \mathrm{~cm}$ with prominent splenic vein and some collaterals at left splenic hilum, and a subsequent colonoscopy was normal and no action was taken at that time.

At 8 weeks gestational age, the patient presented again with left sided abdominal pain and heaviness with radiation to the left shoulder, fatigue and frequent fever. Her platelet and white blood cell (WBC) counts were low (98000/ $\mu \mathrm{L}$ and $2100 / \mu \mathrm{L}$ respectively) and haemoglobin $11 \mathrm{gm} / \mathrm{dL}$. Abdominal ultrasound revealed hepatosplenomegaly with spleen measuring $21 \mathrm{~cm}$ and liver measuring $16.6 \mathrm{~cm}$.

Autoimmune panel and viral serology were negative. No cause for the hepatosplenomegaly was identified. The patient was put on conservative management with repeated ultrasound showing that the hepatosplenomegaly was progressive and reached massive splenomegaly at the third trimester.

The patient underwent elective lower segment caesarean section at 33 weeks, delivering a healthy child.

Post-nataly the patient presented to our hospital with continuing symptoms of pallor, tiredness.

Physical examination revealed pallor and protruded abdomen with tender splenomegaly exceeding the umbilicus, liver span of $18 \mathrm{~cm}$, no chronic liver disease stigmata, and no enlarged lymph nodes.

Laboratory work up showed pancytopenia with WBCs of $1200 / \mu \mathrm{L}, \mathrm{Hb}$ of $7.2 \mathrm{gm} / \mathrm{dl}$ and platelets of $74000 / \mu \mathrm{L}$ with normal kidney functions, ALT 14 unit/L, AST 20 unit/L, total bilirubin 14 micromole per litre, albumin $26 \mathrm{gm} / \mathrm{L}$, INR 1.7 .

Iron saturation $26 \%$, normal $\mathrm{Hb}$ electrophoresis, ESR $20 \mathrm{~mm} / \mathrm{hr}$, LDH 92 unit/L.

Ultrasound of the abdomen revealed progressive hepatosplenomegaly (spleen measuring $33 \mathrm{~cm}$ ) with collaterals around the splenic hilum and small amount of free fluid. Whole body PET CT scan revealed further increasing size of spleen and liver, mild peripheral splenic uptake with indeterminate etiology and no lymphadenopathy (Figure 1).

Hepatitis serology, HIV, malaria smear, (autoimmune hepatitis, leishmaniasis, Wilson's disease) work up, Schistosoma antibodies, EBV \& CMV PCR were all negative.

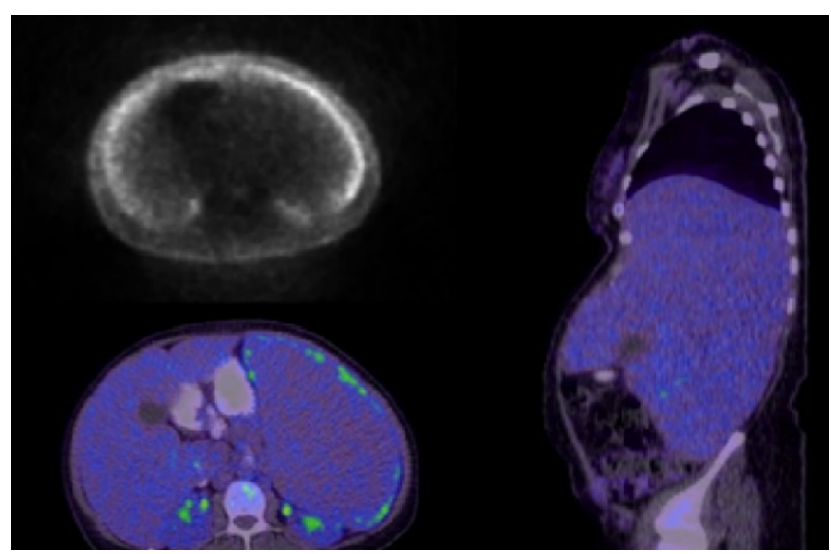

Figure 1: PET CT scan showing hepatosplenomegaly with mild peripheral splenic uptake of indeterminate aetiology, no lymphadenopathy.

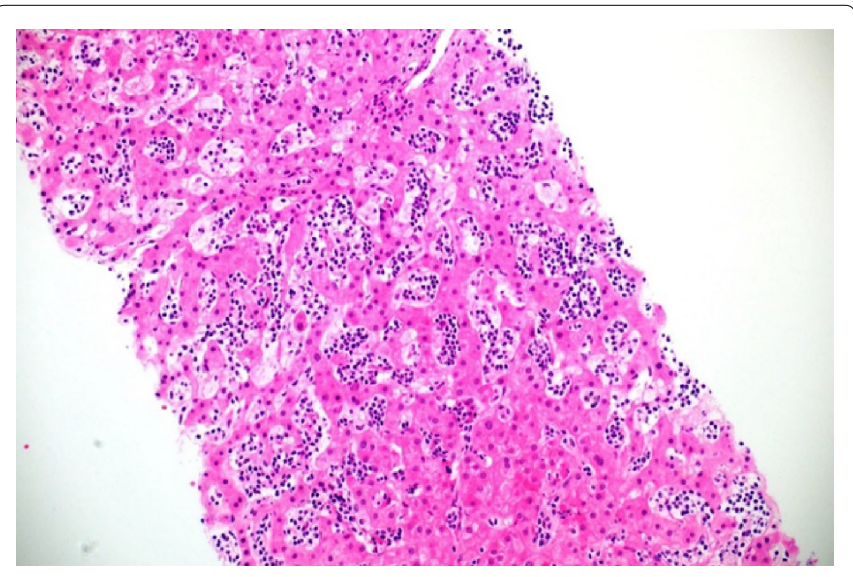

Figure 2: Expansion of the sinusoids by monomorphic infiltrate of small lymphoid cells. Haematoxylin and Eosin X20.

Peripheral smear showed red cells were normochromic with mild anisocytosis, few oval and tear drop cells with increased rouleaux and rare nucleated red blood cells. There was leukopenia and severe neutropenia, majority of the lymphocytes seen were small mature with rare showing irregular nuclear contour. Platelets were markedly reduced in number, cells differential: Neutrophils: $57 \%$, Lymphocytes: 26\%, Monocytes: $16 \%$, Eosinophils: $1 \%$.

Liver biopsy was subsequently performed. The biopsy showed expansion of the hepatic sinusoids by monomorphic infiltrate of small lymphoid cells (Figure 2 and Figure 3). No periportal infiltrates were seen. The intrasinusoidal lymphoid cells were positive for CD2, CD3 (Figure 4) and CD7, confirming the cells to be of T cell origin. Gamma delta immunohistochemistry was also positive (TCR delta partial, weak positive). The cells also co-expressed CD56 (Figure 5) and had a non-activated cytotoxic (TIA-1 positive, granzyme $B$ negative) lymphocytic phenotype. Other markers including CD20, CD5, CD4 and CD8 were negative. The immunophenotypic data, in conjunction with the morphology, supported a diagnosis of $\gamma \delta$ HSGDTL. FISH analysis did not show isochromosome $7 q$ or trisomy 8 . Bone marrow involve- 


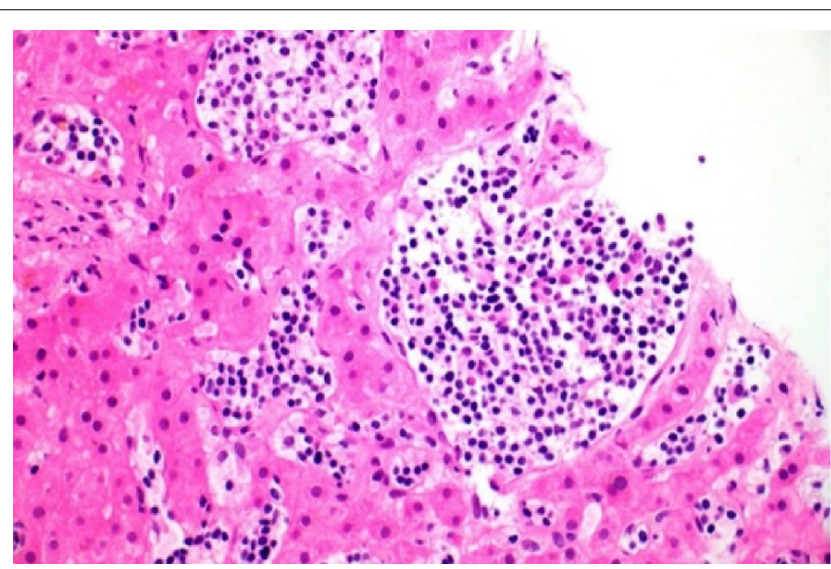

Figure 3: Higher power view showing sinusoidal infiltration of small lymphoid cells. Haematoxylin and Eosin X40.

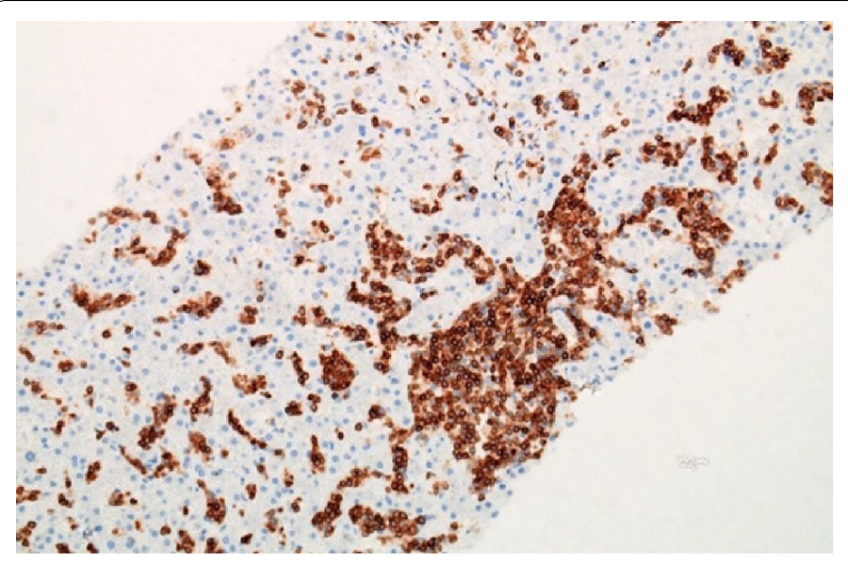

Figure 4: The lymphoid cells are positive for T cell marker, CD3.

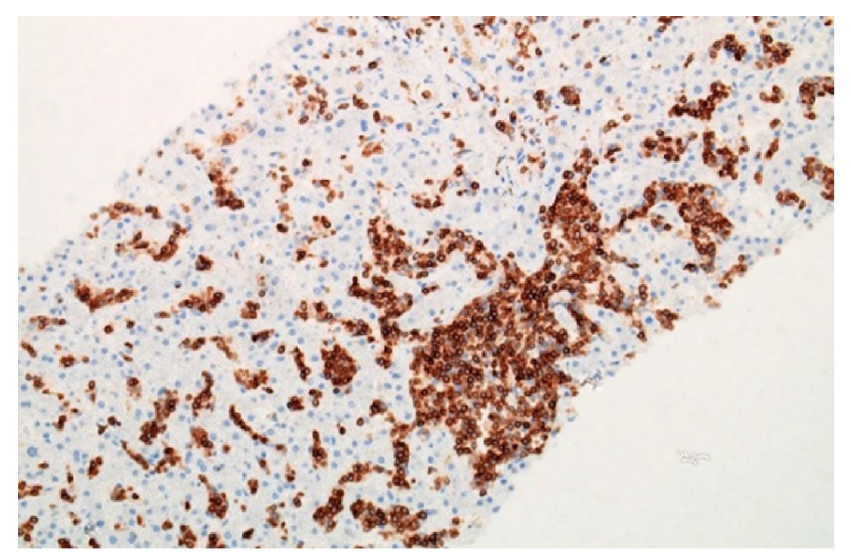

Figure 5: The T cells are also CD56 positive.

ment was also confirmed on biopsy.

Following the diagnosis, the patient received $\mathrm{Cy}$ clophosphamide, Vincristine, Prednisolone (COP) and dexamethasone, (1 cycle) followed by two cycles of Ifosfamide, Carboplatin and Etoposide (ICE) chemotherapy regimens. The general condition of the patient improved and her blood counts steadily increased. Five months after the initial diagnosis, splenectomy was performed, histology of which also showed involvement by $\gamma \delta$ HSGDTL. An allogenic stem cell transplant from Human Leukocyte Antigen (HLA) matched sibling donor was subsequently undertaken. 10 months post-partum, the patient is in clinical remission, with no evidence of disease radiologically or in her bone marrow.

The baby was doing fine at that point.

\section{Discussion}

HSGDTL is a rare Non-Hodgkin's Lymphoma, falling under the category of peripheral T-cell lymphomas. T-lymphocytes identify antigens through polypeptides called $T$ cell receptors that are mainly built by alpha $(\alpha)$ and beta $(\beta)$ chains or rarely $\gamma$ and $\delta$ chains. These receptors undergo rearrangement of their subtypes to produce $\mathrm{CD} 4+/ \mathrm{CD} 8+$ state with $\alpha \beta$ T cell receptor (TCR) on the surface; and CD4-/CD8- state with $\gamma \delta$ TCR on the surface, which in turn are distributed in the lymphoid system with highest concentration in the spleen [2].

The pathogenesis of the transforming events to develop HSGDTL are not well understood, but it was assumed that the triggering event is a monoclonal proliferation with up-regulation of JAK/STAT pathway with or without chromosomal abnormality such as isochromosome $7 q$ trisomy [2].

The majority of HSGDTL express $\gamma \delta$ and a minority have $\alpha \beta$ TCR, which is now considered as a variant of the same disease entity.

The link to long standing immunosuppression has been reported in less than $20 \%$ of the cases of HSGDTL. These cases were either post-transplant patients or were known to have treatment with immunomodulators especially anti-Tumor Necrosis Factor alpha (TNF $\alpha$ ), particularly in patients with Crohn's disease and rheumatoid arthritis [8].

The association between pregnancy and the development of lymphoma has been reported previously [8]. Hodgkin's lymphoma has the most prevalent association with pregnancy. Prognosis of these patients was found to be not too dissimilar from non-pregnant cases. Several changes during pregnancy were theoretically linked to the development of lymphoma, including the immune system changes as a result of high levels of progesterone in the blood.

Cytarabine-based chemotherapy was reported to have better survival rates than alkylating agents and anthracyclines, possibly because of significant expression of P-Glycoprotein (encoded by gene MDR-1) and Glutathione-S-transferases [9].

It was suggested also that trial of demethylating agents in this disease because it was found that A1M1 (a gene might have a role in tumor suppression in HSGDTL) is significantly reduced in HSTL most likely due to promoter methylation [9].

It was also suggested that Syk (a protein thyrosin kinase involved in B-cell receptor signalling and activation and it was found to be common in most peripheral 
T-cell lymphomas) can be an important target for therapy, inhibition of Syk induces apoptosis and blocks proliferation in T-cell lymphoma cell lines [10].

In this report, we wish to highlight a relatively rare lymphoma, hitherto unreported in the Middle East, and presenting during pregnancy as massive hepatosplenomegaly. Recognition of this entity and considering its possibility in the absence of background lymphadenopathy is vital to achieve early diagnosis and treatment.

\section{Authors Contributions}

First three authors contributed equally to this manuscript. Hussam Almasri is the article guarantor.

\section{Financial Disclosure}

Nothing to declare.

\section{Authors Declaration}

Patient consent has been obtained for publication of case details.

\section{References}

1. Swerdlow SH, Campo E, Harris NL, Jaffe ES, Pileri SA, et al. (2008) World Health Organization Classification of Tumours of Haematopoietic and Lymphoid Tissues. IARC Press, Lyon.

2. Yabe M, Miranda RN, Medeiros LJ (2018) Hepatosplenic T-cell Lymphoma: A review of clinicopathologic features, pathogenesis, and prognostic factors. Hum Pathol 74: 5-16.
3. Alonsozana EL, Stamberg J, Kumar D, Jaffe ES, Medeiros LJ, et al. (1997) Isochromosome 7q: The primary cytogenetic abnormality in hepatosplenic gammadelta $\mathrm{T}$ cell lymphoma. Leukemia 11: 1367-1372.

4. Belhadj K, Reyes F, Farcet JP, Tilly H, Bastard C, et al. (2003) Hepatosplenic gammadelta T-cell lymphoma is a rare clinicopathologic entity with poor outcome: Report on a series of 21 patients. Blood 102: 4261-4269.

5. Gumbs AA, Zain J, Neylon E, MacGregor-Cortelli B, Patterson M, et al. (2009) Importance of early splenectomy in patients with hepatosplenic T-cell lymphoma and severe thrombocytopenia, Ann Surg Oncol 16: 2014-2017.

6. Niitsu N, Kohri M, Togano T, Nakamine H, Nakamura S, et al. (2004) Development of hepatosplenic gammadelta T-cell lymphoma with pancytopenia during early pregnancy: A case report and review of the literature. Eur J Haematol 73: $367-371$.

7. Glaser M, Goropevsek A, Kavalar R, Glaser A (2012) Hepatosplenic gamma-delta T-cell lymphoma in a female patient after delivery. Hematol Rep 4: e4.

8. Pohlman B, Macklis RM (2000) Lymphoma and pregnancy. Semin Oncol 27: 657-666.

9. Marion Travert, Yenlin Huang, Laurence de Leval, Nadine Martin-Garcia, Marie-Helene Delfau-Larue, et al. (2012) Molecular features of hepatosplenic T-cell lymphoma unravels potential novel therapeutic targets. Blood 119: 57955806.

10. Feldman AL, Sun DX, Law ME, Novak AJ, Attygalle AD, et al. (2008) Overexpression of Syk tyrosine kinase in peripheral T-cell lymphomas. Leukemia 22: 1139-1143. 